\title{
Title: Capturing the start point of the virus-cell interaction with high-speed 3D single-virus
} tracking

\author{
Authors: Courtney Johnson ${ }^{1} \uparrow$, Jack Exell ${ }^{1} \uparrow$, Yuxin $\operatorname{Lin}^{1}$, Jonathan Aguilar ${ }^{1}$, and Kevin D.
} Welsher ${ }^{1 *}$

\author{
Affiliations: \\ ${ }^{1}$ Department of Chemistry, Duke University; Durham, NC, 27708, USA \\ $\dagger$ These authors contributed equally to this work. \\ *Corresponding author. Email: kdw32@duke.edu
}

Abstract: The early stages of the virus-cell interaction have long evaded observation by existing microscopy methods due to the rapid diffusion of virions in the extracellular space and the large 3D cellular structures involved. Here we present an active-feedback single-virus tracking method with simultaneous volumetric imaging of the live cell environment to address this knowledge gap to present unprecedented detail to the extracellular phase of the infectious cycle. We report previously unobserved phenomena in the early stages of the virus-cell interaction, including skimming contact events at the millisecond timescale, orders of magnitude change in diffusion coefficient upon binding, and cylindrical and linear diffusion modes along filopodia. Finally, we demonstrate how this new method can move single-virus tracking from simple monolayer culture towards more tissue-like conditions by tracking single virions in tightly packed epithelial cells. This multi-resolution method presents new opportunities for capturing fast, 3D processes in biological systems.

One-Sentence Summary: Active-feedback 3D single-virus tracking enables an unprecedented look at the early stages of virus-cell interactions.

\section{Main Text:}

The ongoing SARS-CoV-2 pandemic has demonstrated with frightening clarity the need for fundamental research in physical virology to exploit and counter the mechanisms of viral infection. Single-virus tracking (SVT) methods have emerged as a powerful tool in our understanding of viral infection (1-3). These methods have uncovered virion binding mechanisms to the cell surface $(4,5)$, distinguished internalization pathways (6-10), identified the cellular location of envelope fusion (11-17), characterized cytoskeletal trafficking $(5,10-13,18-24)$, and demonstrated how viruses hijack filopodia to efficiently infect neighboring cells $(10,14,25-30)$.

Despite these advances, it has thus far not been possible to follow virions starting in the extracellular space with sufficient detail to interrogate this important phase of viral infection. The first point of contact with the host organism occurs in the extracellular space of the epithelia, whose cells form a tightly packed arrangement protected by an extended mucus layer and glycocalyx (3135). The structure of the extracellular matrix (ECM) has been shown to be critical to the viral lifecycle, undergoing changes in structure and composition upon introduction of viral pathogens (36) and hosting biofilm-like viral assemblies for cell-to-cell transmission (37). Unfortunately, current live-cell virus tracking methods cannot probe deep within samples to visualize extracellular dynamics without sacrificing temporal resolution.

Conventionally applied image-based SVT methods suffer from limitations caused by attempting to track and image disparately scaled objects on one platform simultaneously. Total internal reflection fluorescence (TIRF) methods are restricted to areas near the coverslip surface. 
Confocal methods acquire volumetric images over timescales too slow to measure single virion dynamics in the extracellular space. Even with the faster imaging rates of light sheet methods (38, 39 ), volumetric data is still acquired frame-by-frame. Consequently, the temporal resolution scales poorly as the axial extent of the process in question grows. Active-feedback tracking methods seek to overcome the low temporal resolution of image-based tracking methods by focusing exclusively on the tracked particle (40). Such methods can localize particles with high spatial and temporal resolution but are unable to acquire information about the surrounding cellular context. There are similar drawbacks for interferometric scattering (iScat) microscopy, which has been used to track membrane-bound viruses $(41,42)$ but lacks the depth range and environmental context needed to probe the extracellular phase.

Instead of a single microscope limited by the sacrifices to speed, spatial resolution, or axial depth required when imaging disparate scales, a new, multi-resolution approach is needed, wherein viral dynamics are collected with millisecond or better temporal resolution and complemented by high-resolution volumetric images of the critical cellular context. To date, no such comprehensive method exists.

To address this gap, we present 3D Tracking and Imaging Microscopy (3D-TrIm), a multimodal approach that integrates a real-time active-feedback tracking microscope (43-45) with a volumetric imaging system (46) capable of simultaneously tracking the high-speed dynamics of extracellular virions while imaging the surrounding 3D, live-cell environment (Fig. 1A). This multi-resolution approach provides unprecedented glimpses into multiple stages of the virus-cell interaction. Using 3D-TrIm, we acquired over 3000 viral trajectories and quantified their dynamics as they interacted with the cell in four distinct phases: Extracellular, surface-bound, surface mobile, and intracellular. Finally, 3D-TrIm was applied to advance SVT from monolayer cell culture to tightly packed, 3D epithelial culture models, providing a new window into this critical stage of viral infection.

\section{D-TrIm Overview}

Capturing extracellular viral dynamics requires the ability to track a rapidly diffusing target in three dimensions. To accomplish this, we apply the recently developed 3D single-molecule active real-time tracking (3D-SMART) $(43,44)$. 3D-SMART is an active-feedback microscopy method that uses real-time position information to "lock on" to moving fluorescent targets. Critically, 3D-SMART can capture particles diffusing at up to $10 \mathrm{~mm}^{2} / \mathrm{s}$ with only a single fluorophore label (43), making it an ideal choice for capturing diffusing virions (45).

The implementation of 3D-SMART in the current work is similar to previous reports. Briefly, a rapid 3D laser scan excites photons from the fluorescently labeled diffusing viral particle (Fig. $1 \mathrm{~B}$, movie S1). Photon arrival times collected on a single-photon counting avalanche photodiode (SPCM-APD) are used to calculate the real-time position. The real-time position is then used in an integral feedback loop to move the sample via a piezoelectric stage, effectively fixing the moving target in the focus of the microscope objective, even when highly mobile in three dimensions. 3D-SMART produces a three-dimensional particle trajectory with temporal resolution of $1 \mathrm{msec}$ or better, limited only by the photon count rate, with localization precision up to $\sim 20$ $\mathrm{nm}$ in $\mathrm{XY}$ and $\sim 80 \mathrm{~nm}$ in $\mathrm{Z}$ (fig. S1) and duration only limited by the travel range of the piezoelectric stage $(75 \mu \mathrm{m} \times 75 \mu \mathrm{m} \times 50 \mu \mathrm{m}$; XYZ).

While 3D-SMART is ideally suited for capturing the fast dynamics of extracellular viral particles over vast axial ranges, it lacks environmental context. A complimentary rapid volumetric imaging method is needed to capture the live-cell environment. The choice of imaging system is non-trivial as the active-feedback nature of 3D-SMART requires a moving stage which places two constraints that must be considered. First, imaging with camera-based methods would result in 
motion blur across a single exposure time. Second, the stage cannot be stepped to perform "zstacking" for volumetric imaging.

To meet these criteria, we implement 3D Fast Acquisition by z-Translating Raster (3DFASTR) microscopy to provide rapid volumetric imaging around the tracked viral particle (46). 3D-FASTR uses a two-photon laser scanning microscope outfitted with an electrically tunable lens (ETL) to translate the imaging laser focus optically. The short pixel dwell time $(\sim 1 \mu \mathrm{sec})$ prevents motion blur, and the ETL enables 3D imaging without translating the sample. At optimized scan frequencies, the combination of the $\mathrm{XY}$ raster scan and the $\mathrm{Z}$ focal scan evenly samples voxels in a tessellating pattern which accumulates to fill the entire volume (Fig. 1C, movie S1). At short acquisition times, unsampled voxels will have many sampled neighbors such that the volumetric imaging rate can be increased 2- to 4-fold over conventional serial frame acquisition methods through interpolation (46). The ability to rapidly image volumes without moving the sample makes 3D-FASTR the perfect complement to 3D-SMART.

Excitation and emission for both tracking and imaging are routed through a single shared objective lens $(100 \times, \mathrm{NA}=1.4)$ on an inverted microscope stand outfitted with a $3 \mathrm{D}$ piezoelectric stage. The piezoelectric stage is mounted on a secondary motorized stage that remains static during acquisition but can be moved between trajectories to capture different areas of the sample. (fig. S2). Registration of the 3D-SMART and 3D-FASTR systems is required to generate combined 3D-TrIm datasets (fig. S3-4). The piezoelectric stage represents a shared spatial grid between the two microscopes; the real-time stage position is a measure of the tracked particle's location that is read out every $10 \mu \mathrm{sec}$ and tagged to the imaging XYZ voxel position. These relative positions are then used with calibration information (fig. S5-6) to place each image voxel in the shared global volume space. In 3D-TrIm, these voxels can be integrated over an entire trajectory to form a complete volume or sampled at higher rates (as fast as $\sim 4 \mathrm{sec} /$ volume) to construct volume sequences over the course of a single trajectory (Fig. 1D).

For this pioneering study into the early stages of the virus-cell interaction, we investigated the journey of vesicular stomatitis virus (VSV)-G-pseudotyped lentiviruses from the extracellular solution to the cell surface and beyond. We chose this specific virus-like particle (VLP) due to its wide cellular tropism. A behavior attributed to the ubiquitous host entry partner of VSV-G, the low-density lipoprotein receptor (LDLR) (47), which has established VSV-G as the standard for gene delivery (48). We utilized an efficient and well-characterized internal virus labeling approach whereby fluorescent proteins fused to the HIV-1 viral protein R (Vpr) are packaged into budding lentiviruses (49). Successful incorporation of eGFP.Vpr into single virions was confirmed by tracking VSV-G eGFP.Vpr in the absence of cells and by immunofluorescence experiments (fig. S7-9). Critically, these VLPs were shown to remain infective (fig. S10).

\section{D-TrIm reveals that the extracellular dynamics of virions are cell-type and distance- dependent}

To initiate data collection, the 3D-TrIm microscope locates diffusive virions by searching a plane approximately $5 \mu \mathrm{m}$ above the apical cell surface so that viruses are captured prior to viruscell first contacts. When a VLP enters the 3D-SMART tracking volume, a burst of photons is observed on the single-photon counter, which triggers the active-feedback tracking loop and 3DFASTR image acquisition. An example trajectory of these early interactions is shown in Fig. 2AC, which features live HeLa cells fluorescently labeled with the nucleic acid stain SYTO61 (additional examples: fig. S14-18). Taking advantage of the co-registered individual local volumes (fig. S11), a global 3D render of the cells was constructed using the maximum intensity of each voxel over time. The viral trajectory is constructed from the piezoelectric stage coordinates, recorded every $10 \mu \mathrm{sec}$, and shown here at $3 \mathrm{msec}$ sampling intervals. The single VSV-G VLP 
was initially captured in the search plane and diffused for $\sim 90$ seconds, reaching a maximum height of nearly $20 \mu \mathrm{m}$ above the cell surface. The virion closely approaches and intermittently touches the surface of several cells over the course of the trajectory.

These periods of transient contact ("skimming") were quantified in two ways. First, changepoint analysis was applied to identify changes in the diffusive state of the VLP as it traverses the volume $(50,51)$. The extracted diffusion coefficients are shown as color-coded lines in Fig. 2D. Second, the distance between the VLP and the cell surface was calculated using segmented volume data (fig. S12). This VLP-cell distance is visualized in Fig. 2B, a color-mapped volume with yellow/red indicating approach within $1 \mu \mathrm{m} / 0.5 \mu \mathrm{m}$ (comparable to the axial extent of the 3D-FASTR point-spread function, fig. S13), respectively. The trajectory reveals multiple and repeated contact events at various surface locations (highlighted in purple in Fig. 2D). While the HeLa cell shown is LDLR positive, these skimming events are not restricted to receptor-positive cells but are also observed in GM701 fibroblasts (low LDLR expression level) (Fig. 2F-I and movie S2).

These transient contacts can be quantified to yield their occurrence (fig. S19A), frequency (fig. S19B), and dwell time (fig. S20) for each cell type. These contacts can be as short as several milliseconds in duration but numerous per trajectory, without promoting long-duration viral attachment. Exponential fitting of contact event durations on HeLa cells yielded two populations with dwell times of $23 \pm 1$ and $102 \pm 5 \mathrm{msec}$ (fig. S20A). The shorter dwell time is consistent with free diffusion, while the longer dwell time indicates an interaction of the VLP with the cell surface or extracellular matrix. These dwell times were longer for HeLa $(102 \pm 5 \mathrm{msec})$ compared to the low-LDLR GM701 fibroblast cells ( $88 \pm 8 \mathrm{msec}$, fig. S20B), though they exhibited identical fast components. Experiments on a positive control fibroblast cell line with high LDLR expression (BJ) revealed that these longer dwell times are likely not receptor related but potentially related to cell morphology (fig. S20C).

The increased dwell time near the cell surface also correlated with slower diffusion in the presence of HeLa cells. A trajectory-wide analysis of "skimming" VSV-G VLPs showed 80\% of trajectories acquired in the presence of HeLa cells have diffusion coefficients within the range of 0.5 to $3.4 \mu \mathrm{m}^{2} / \mathrm{s}$ (fig. S21), consistent with diffusive rates in the presence of cells in previous reports $(4,5,51)$. Interestingly, VLPs exhibited increased diffusivity of 1.3 to $4.5 \mu \mathrm{m}^{2} / \mathrm{s}$ for thinner fibroblast cells, revealing a cell-type dependence on extracellular diffusivity (Fig. $2 \mathrm{~K}, t$-test, $\mathrm{p}<$ 0.01). The unique correlative imaging and high-speed tracking capabilities of the 3D-TrIm microscope enabled a quantitative examination of the relationship between VLP diffusion and distance from the cell surface. This correlation analysis revealed a distance-dependent relationship, with VLPs slowing down as they near the cell surface. Statistically-significant inhibition of diffusion was observed within $2.5 \mu \mathrm{m}$ of the cell surface for all cell types tested (fig. S21). Again, compared to the fibroblasts, VLPs in the presence of HeLa trended towards lower diffusion coefficients near the cell surface (fig. S21). This difference again suggests a morphological effects imposed by the more irregular shaped HeLa compared to flatter, more uniform fibroblasts.

$40 \quad$ While all three cell types showed a trend towards slower diffusion near the cell surface, each still displayed a significant fraction of VLPs with segments of diffusion $>1 \mu \mathrm{m}^{2} / \mathrm{s}$ at less than 1 $\mu \mathrm{m}$ from the cell surface. Again, this effect was cell-type dependent, with BJ cells showing a much higher percentage of fast diffusing VLPs near the surface (BJ: 33.6\% $\pm 11.8 \%$, HeLa: 16.8\% \pm $3.9 \%$, GM701; $16.7 \% \pm 7.3 \%$ ). This fast diffusion near the cell surface suggests a complex interplay between the extracellular environment and VLP dynamics.

\section{Long duration viral binding events}


In addition to the transient contacts observed above, long-term binding events of individual VSV-G VLPs on the cell surface were also captured by 3D-TrIm. Unlike skimming, these binding events display dramatic diffusivity changes upon contact. In the first example (Fig. 3A-D, fig. S22, and movie S3), the virus initially undergoes several skimming events, marked by close approach to the surface of multiple cells with subtle changes in the diffusion coefficient. At $\sim 70 \mathrm{~s}$, as the virus-cell distance reaches a minimum, viral diffusivity drops by two orders of magnitude and remains bound for several minutes (indicated by the pink box in Fig. 3D). It was also observed that bound viral particles detach after landing and diffuse away or even bind again elsewhere. Fig. 3E-G shows a VLP initially bound between two protrusions on an actin-stained GM701 fibroblast (see also fig. S23, movie S4). This bound state (D $\sim 0.04 \mu \mathrm{m}^{2} / \mathrm{s}$, Fig. 3E, yellow) persists for $\sim 23$ sec, before detaching to free diffusion ( $D>2 \mu \mathrm{m}^{2} / \mathrm{s}$ ). After only $\sim 4 \mathrm{sec}$ of free diffusion, viral diffusivity drops to less than $0.1 \mu \mathrm{m}^{2} / \mathrm{s}$ upon contact and remains bound for several seconds before detaching again to free diffusion and ultimately leaving the trackable volume (additional example: fig. S24). Combined with the msec-scale dwell times above, these multiple and long-term binding events suggest that cell-VLP attachment events cover a large range of timescales.

\section{VLPs exhibit distinct diffusional modes on actin-rich protrusions}

Beyond the skimming and bound states described above, we observed a spectrum of dynamic interactions between VLPs and the cell surface. One frequent example was the interaction between VLPs and actin-based protrusions on the cell membrane. Using 3D-TrIm, two distinct classes of interactions between VLPs and live-cell filopodia were observed. First, we observed viral particles binding directly to these structures from the extracellular space (Fig. 4A-E, fig. S25 and movie S5). While not stained and therefore not visible in the image, the virus's path along the surface creates a high-resolution map, carving out the nanoscale cylindrical morphology of the protrusion surface (Fig. 4D, E). Similar to how super-localization of emitters enables nanoscale resolution of cellular structures in super-resolution methods, the high 3D precision and rapid sampling of 3DSMART turns the virus into a nanoscale pen that draws out local features. The feature traced by this VLP protrudes $\sim 1 \mu \mathrm{m}$ vertically from the cell surface (Fig. 4D). A best-fit cylinder gives a radius of $105 \pm 8.4 \mathrm{~nm}$ (Fig. 4E), consistent with the observed size of filopodial protrusions, and notably, demonstrating the ability for 3D-TrIm to super-resolve tracked features beyond the diffraction limit. These structures were dynamic and resulted in changing cylindrical structure within the viral trajectory over time (Fig. 4F-I), with radii tapering for more distal parts of the filopodium structure. VLPs on these structures were consistently measured to have diffusivity values $\sim 0.01 \mu \mathrm{m}^{2} / \mathrm{s}$ (fig. S25-S27), nearly identical to other types of membrane diffusion observed (fig. S31).

In contrast, linear "surfing" $(52,53)$ along the axis of larger filopodia was also detected (Fig. 4J-L, fig. S29-30). Fig. 4J presents a top-down view of a 3D-TrIm volume, showing linear VLP motion along the contour of a filament. The simultaneously collected volumetric image views show that the VLP "surfs" along the top surface of the actin-rich protrusion (Fig. 4K-L, fig. S2930). The VLP exhibits two distinct diffusive regimes: one consistent with a bound particle and a second associated with rapid directed motion along the protrusion. Changepoint analysis (Fig. 4K) shows that the VLP starts in a bound state $\left(0.0042 \pm 0.0011 \mu \mathrm{m}^{2} / \mathrm{s}\right)$, followed by a rapid change in diffusion to a rate up to two orders of magnitude greater $\left(0.61 \pm 0.15 \mu \mathrm{m}^{2} / \mathrm{s}\right)$ as the virus undergoes linear displacement along the filopodia shaft, before again returning to a quasi-bound state $\sim 7 \mu \mathrm{m}$ from its previous position. As observed in the render, these points of increased diffusion correlate with higher-intensity regions that indicate a greater concentration of f-actin. Interestingly, the size of the cellular protrusion seemed to affect the viral diffusivity behavior on the membrane. Smaller 
structures that could not be resolved by the imaging alone show cylindrical diffusion whereas linear surfing was prominent on much larger, adherent f-actin structures.

\section{Single viral dynamics in the epithelia: beyond monolayer cell culture models}

As is typical of SVT experiments, the previous examples were captured in the presence of monolayer cultures, which have been shown to differ from more realistic tissue models (54). Here, we demonstrate the potential for 3D-TrIm to operate in complex environments at considerable depths in systems that more closely approximate the infection routes of viruses in vivo. A system of particular relevance to the extracellular dynamics of viruses (and to respiratory viruses in particular) is the epithelia, which is protected by a thick mucus layer $(31,32)$ and a size-excluding pericilliary layer (PCL) (55). For well-differentiated epithelial cells to form tightly packed arrangements in vitro, they must be grown on a semipermeable membrane support to allow access to the basolateral layer and provide a more realistic growth environment. The thick $(>10 \mu \mathrm{m})$, tightly-packed epithelial layer cannot be grown directly on a coverslip, making observation of dynamics in these critical systems impossible in conventional microscopy methods. In contrast, 3D-TrIm's large axial range enables unprecedented high-speed single-virus tracking in these more biologically relevant tissue models.

Epithelial model systems were prepared by growing HT29-MTX cells on a semipermeable membrane support and inverted so that the cells were suspended above the coverslip surface (fig. $\mathrm{S} 32$ ). The diffusion of viruses into this tightly packed layer can be captured from this vantage with msec temporal resolution (Fig. 5A-D, fig. S33-34 and movie S6). The average diffusion coefficient for VSV-G traveling in this complex environment is $1.29 \pm 0.44 \mu \mathrm{m}^{2} / \mathrm{s}$, close to that of freely diffusing virus particles (fig. S9), suggesting the extracellular matrix does not present a significant physical barrier. However, despite the fast diffusion, the VLP remains confined near the surface of multiple cells for nearly 3 minutes. This rapid but trapped diffusion is in stark contrast to viruses near monolayer cultured cells, where the rapid diffusion limits the dwell time near the cell surface. Notably, this example demonstrates the capability for 3D-TrIm to track at low signal levels $(35 \mathrm{kHz})$ in complex environments, which is a significant advantage over other prior active feedback tracking methods, which require several hundred $\mathrm{kHz}$ for successful tracking $(51,56)$.

\section{Discussion / Conclusion}

There are several features of 3D-TrIm that make it extendable to future studies. First, not restricted to capturing rapid extracellular dynamics, 3D-TrIm's high-resolution tracking ability extends to the later, internalized stages of the infectious cycle (movie S7 and fig. S35-36). These data demonstrate that the VLPs in this study exhibit the hallmarks of infectious virions in viable cells, undergoing normal cellular processes like endocytosis and intracellular trafficking. 3D-TrIm is fully compatible with live-cell studies and active biological processes.

Second, 3D-TrIm can observe single-particle dynamics within the same area over long periods to expand beyond the single-particle nature intrinsic to active-feedback tracking methods (40). Such prolonged live-cell imaging is made possible by a combination of continual laser scanning and viral motion, which reduces the overall laser dwell time at any single position. Multiple-trajectory registration enables 3D-TrIm to accumulate a population of trajectories sufficient to perform statistical analyses on the dynamics and activity of virions in a single area (Fig. 6A-C, fig. S37 and movie S8). We demonstrate this capability in Fig. 6B, which shows the population average (27 of 38 trajectories) of VLP position and diffusivity, revealing different VLPs frequent similar areas of the cell surface and extracellular matrix. Further experiments may uncover what factors influence the heterogenous dwell time of virions to these particular regions of the surface. 
The data collected here by the 3D-TrIm microscope represent a dramatic step forward for SVT at high speeds in complex systems. Prior to this study, the immobilization and detachment behaviors of single virions could be observed by epifluorescence microscopy $(4,57)$. However, here 3D-TrIm gives a continuous and uninterrupted timeline of the viral particle through the entire process, complete with volumetric imaging and environmentally contextualized diffusion analysis. The skimming events observed here are the first of their kind and demonstrate how cell morphology can affect viral diffusion and how diffusion is inhibited at the cell surface. For membrane-bound VLPs, while the phenomena of viruses utilizing actin-rich protrusions as tracks to facilitate transport along the plasma membrane has been well-described $(10,14,25-30)$, the distinct transport modes revealed here give insight into the different ways that VLPs interact with actin-rich protrusions. Finally, we demonstrated never before reported rapid diffusion of single VLPs in a tightly packed epithelial layer, paving the way towards high-speed SVT in more realistic biological systems. Future studies with 3D-TrIm will be able to probe various unanswered questions in viral dynamics, including the "molecular walker" hypothesis (58) and the seeming impenetrability of the PCL for particles larger than $40 \mathrm{~nm}$ in diameter (55). Importantly, the application of this new technique can be extended to any system where fast dynamics of nanoscale objects occur over large volumetric scales, including delivery of nanoscale drug candidates to the lungs (59) and through leaky tumor vasculature (60).

\section{References and Notes}

1. B. Brandenburg, X. Zhuang, Virus trafficking - learning from single-virus tracking. Nat Rev Microbiol 5, 197-208 (2007).

2. S. L. Liu et al., Single-Virus Tracking: From Imaging Methodologies to Virological Applications. Chem Rev 120, 1936-1979 (2020).

3. N. Parveen, D. Borrenberghs, S. Rocha, J. Hendrix, Single Viruses on the Fluorescence Microscope: Imaging Molecular Mobility, Interactions and Structure Sheds New Light on Viral Replication. Viruses 10, (2018).

4. T. Endress et al., HIV-1-cellular interactions analyzed by single virus tracing. Eur Biophys J 37, 1291-1301 (2008).

5. G. Seisenberger et al., Real-time single-molecule imaging of the infection pathway of an adeno-associated virus. Science 294, 1929-1932 (2001).

6. M. J. Rust, M. Lakadamyali, F. Zhang, X. Zhuang, Assembly of endocytic machinery around individual influenza viruses during viral entry. Nat Struct Mol Biol 11, 567-573 (2004).

7. L. Pelkmans, J. Kartenbeck, A. Helenius, Caveolar endocytosis of simian virus 40 reveals a new two-step vesicular-transport pathway to the ER. Nat Cell Biol 3, 473-483 (2001).

8. D. K. Cureton, C. E. Harbison, E. Cocucci, C. R. Parrish, T. Kirchhausen, Limited transferrin receptor clustering allows rapid diffusion of canine parvovirus into clathrin endocytic structures. J Virol 86, 5330-5340 (2012).

9. E. Z. Sun et al., Real-Time Dissection of Distinct Dynamin-Dependent Endocytic Routes of Influenza A Virus by Quantum Dot-Based Single-Virus Tracking. ACS Nano 11, 4395-4406 (2017).

10. H. Xu et al., Real-time Imaging of Rabies Virus Entry into Living Vero cells. Sci Rep 5, 11753 (2015).

11. M. Lakadamyali, M. J. Rust, H. P. Babcock, X. Zhuang, Visualizing infection of individual influenza viruses. Proc Natl Acad Sci U S A 100, 9280-9285 (2003). 
12. H. M. van der Schaar et al., Dissecting the cell entry pathway of dengue virus by singleparticle tracking in living cells. PLoS Pathog 4, e1000244 (2008).

13. H. M. van der Schaar et al., Characterization of the early events in dengue virus cell entry by biochemical assays and single-virus tracking. J Virol 81, 12019-12028 (2007).

14. K. E. Coller et al., RNA interference and single particle tracking analysis of hepatitis $\mathrm{C}$ virus endocytosis. PLoS Pathog 5, e1000702 (2009).

15. C. Qin et al., Real-time dissection of dynamic uncoating of individual influenza viruses. Proc Natl Acad Sci U S A 116, 2577-2582 (2019).

16. Q. Li et al., Single-Particle Tracking of Human Immunodeficiency Virus Type 1 Productive Entry into Human Primary Macrophages. ACS Nano 11, 3890-3903 (2017).

17. H. Ewers et al., Single-particle tracking of murine polyoma virus-like particles on live cells and artificial membranes. Proc Natl Acad Sci U S A 102, 15110-15115 (2005).

18. M. Suomalainen et al., Microtubule-dependent plus- and minus end-directed motilities are competing processes for nuclear targeting of adenovirus. J Cell Biol 144, 657-672 (1999).

19. D. McDonald et al., Visualization of the intracellular behavior of HIV in living cells. $J$ Cell Biol 159, 441-452 (2002).

20. C. J. Bailey, R. G. Crystal, P. L. Leopold, Association of adenovirus with the microtubule organizing center. J Virol 77, 13275-13287 (2003).

21. N. Arhel et al., Quantitative four-dimensional tracking of cytoplasmic and nuclear HIV-1 complexes. Nat Methods 3, 817-824 (2006).

22. S. L. Liu et al., Effectively and efficiently dissecting the infection of influenza virus by quantum-dot-based single-particle tracking. ACS Nano 6, 141-150 (2012).

23. L. J. Zhang et al., A "Driver Switchover" Mechanism of Influenza Virus Transport from Microfilaments to Microtubules. ACS Nano 12, 474-484 (2018).

24. Q. M. Wu et al., Uncovering the Rab5-Independent Autophagic Trafficking of Influenza A Virus by Quantum-Dot-Based Single-Virus Tracking. Small 14, e1702841 (2018).

25. N. M. Sherer et al., Retroviruses can establish filopodial bridges for efficient cell-to-cell transmission. Nat Cell Biol 9, 310-315 (2007).

26. R. Dixit, V. Tiwari, D. Shukla, Herpes simplex virus type 1 induces filopodia in differentiated P19 neural cells to facilitate viral spread. Neurosci Lett 440, 113-118 (2008).

27. M. Schelhaas et al., Human papillomavirus type 16 entry: retrograde cell surface transport along actin-rich protrusions. PLoS Pathog 4, e1000148 (2008).

28. S. Sowinski et al., Membrane nanotubes physically connect T cells over long distances presenting a novel route for HIV-1 transmission. Nat Cell Biol 10, 211-219 (2008).

29. J. Mercer, A. Helenius, Vaccinia virus uses macropinocytosis and apoptotic mimicry to enter host cells. Science 320, 531-535 (2008).

30. J. Jose, J. Tang, A. B. Taylor, T. S. Baker, R. J. Kuhn, Fluorescent Protein-Tagged Sindbis Virus E2 Glycoprotein Allows Single Particle Analysis of Virus Budding from Live Cells. Viruses 7, 6182-6199 (2015).

31. S. C. Baos, D. B. Phillips, L. Wildling, T. J. McMaster, M. Berry, Distribution of sialic acids on mucins and gels: a defense mechanism. Biophys $J$ 102, 176-184 (2012).

32. M. Kesimer et al., Molecular organization of the mucins and glycocalyx underlying mucus transport over mucosal surfaces of the airways. Mucosal Immunol 6, 379-392 (2013).

33. S. K. Linden, P. Sutton, N. G. Karlsson, V. Korolik, M. A. McGuckin, Mucins in the mucosal barrier to infection. Mucosal Immunol 1, 183-197 (2008). 
34. C. Ridley, D. J. Thornton, Mucins: the frontline defence of the lung. Biochem Soc Trans 46, 1099-1106 (2018).

35. D. J. Thornton, K. Rousseau, M. A. McGuckin, Structure and function of the polymeric mucins in airways mucus. Annu Rev Physiol 70, 459-486 (2008).

36. H. Tomlin, A. M. Piccinini, A complex interplay between the extracellular matrix and the innate immune response to microbial pathogens. Immunology 155, 186-201 (2018).

37. A.-M. Pais-Correia et al., Biofilm-like extracellular viral assemblies mediate HTLV-1 cell-to-cell transmission at virological synapses. Nature Medicine 16, 83-89 (2010).

38. L. Gao, L. Shao, B.-C. Chen, E. Betzig, 3D live fluorescence imaging of cellular dynamics using Bessel beam plane illumination microscopy. Nat. Protocols 9, 1083-1101 (2014).

39. T. A. Planchon et al., Rapid three-dimensional isotropic imaging of living cells using Bessel beam plane illumination. Nature Methods 8, 417 (2011).

40. S. Hou, C. Johnson, K. Welsher, Real-Time 3D Single Particle Tracking: Towards Active Feedback Single Molecule Spectroscopy in Live Cells. Molecules 24, 2826 (2019).

41. Y. F. Huang et al., Coherent Brightfield Microscopy Provides the Spatiotemporal Resolution To Study Early Stage Viral Infection in Live Cells. ACS Nano 11, 2575-2585 (2017).

42. R. W. Taylor, V. Sandoghdar, Interferometric Scattering Microscopy: Seeing Single Nanoparticles and Molecules via Rayleigh Scattering. Nano Lett 19, 4827-4835 (2019).

43. S. Hou, J. Exell, K. Welsher, Real-time 3D single molecule tracking. Nature Communications 11, 3607 (2020).

44. S. Hou, X. Lang, K. Welsher, Robust real-time 3D single-particle tracking using a dynamically moving laser spot. Optics Letters 42, 2390-2393 (2017).

45. S. Hou, K. Welsher, An Adaptive Real-Time 3D Single Particle Tracking Method for Monitoring Viral First Contacts. Small 15, e1903039 (2019).

46. C. Johnson, J. Exell, J. Kuo, K. Welsher, Continuous focal translation enhances rate of point-scan volumetric microscopy. Opt. Express 27, 36241-36258 (2019).

47. D. Finkelshtein, A. Werman, D. Novick, S. Barak, M. Rubinstein, LDL receptor and its family members serve as the cellular receptors for vesicular stomatitis virus. Proceedings of the National Academy of Sciences 110, 7306-7311 (2013).

48. L. Naldini et al., In Vivo Gene Delivery and Stable Transduction of Nondividing Cells by a Lentiviral Vector. Science 272, 263-267 (1996).

49. T. M. Desai et al., Fluorescent protein-tagged Vpr dissociates from HIV-1 core after viral fusion and rapidly enters the cell nucleus. Retrovirology 12, 88 (2015).

50. D. Montiel, H. Cang, H. Yang, Quantitative Characterization of Changes in Dynamical Behavior for Single-Particle Tracking Studies. Journal of Physical Chemistry B 110, 19763-19770 (2006).

51. K. Welsher, H. Yang, Multi-resolution 3D visualization of the early stages of cellular uptake of peptide-coated nanoparticles. Nat Nanotechnol 9, 198-203 (2014).

52. M. J. Lehmann, N. M. Sherer, C. B. Marks, M. Pypaert, W. Mothes, Actin- and myosindriven movement of viruses along filopodia precedes their entry into cells. J Cell Biol 170, 317-325 (2005).

53. W. Mothes, N. M. Sherer, J. Jin, P. Zhong, Virus cell-to-cell transmission. J Virol 84, 8360-8368 (2010).

54. C. Jensen, Y. Teng, Is It Time to Start Transitioning From 2D to 3D Cell Culture?

Frontiers in Molecular Biosciences 7, (2020). 
55. B. Button et al., A periciliary brush promotes the lung health by separating the mucus layer from airway epithelia. Science 337, 937-941 (2012).

56. E. P. Perillo et al., Deep and high-resolution three-dimensional tracking of single particles using nonlinear and multiplexed illumination. Nature Communications $\mathbf{6}$, (2015).

57. W. Nasir, M. Bally, V. P. Zhdanov, G. Larson, F. Höök, Interaction of Virus-Like Particles with Vesicles Containing Glycolipids: Kinetics of Detachment. J Phys Chem B 119, 11466-11472 (2015).

58. P. H. E. Hamming, N. J. Overeem, J. Huskens, Influenza as a molecular walker. Chem Sci 11, 27-36 (2020).

59. C. M. Bobba et al., Nanoparticle delivery of microRNA-146a regulates mechanotransduction in lung macrophages and mitigates injury during mechanical ventilation. Nature Communications 12, 289 (2021).

60. D. Sun, S. Zhou, W. Gao, What Went Wrong with Anticancer Nanomedicine Design and How to Make It Right. ACS Nano 14, 12281-12290 (2020).

61. Q. Wang, W. E. Moerner, Optimal strategy for trapping single fluorescent molecules in solution using the ABEL trap. Applied Physics B 99, 23-30 (2010).

62. A. Mermillod-Blondin, E. McLeod, C. B. Arnold, High-speed varifocal imaging with a tunable acoustic gradient index of refraction lens. Optics Letters 33, 2146-2148 (2008).

63. A. P. Fields, A. E. Cohen, Optimal tracking of a Brownian particle. Optics Express 20, 22585-22601 (2012).

64. D. Garcia, Robust smoothing of gridded data in one and higher dimensions with missing values. Comput Stat Data Anal 54, 1167-1178 (2010).

65. Y. Wakabayashi, J. Chua, J. M. Larkin, J. Lippincott-Schwartz, I. M. Arias, Fourdimensional imaging of filter-grown polarized epithelial cells. Histochem Cell Biol 127, 463-472 (2007).

66. P. I. Ortinski, B. O'Donovan, X. Dong, B. Kantor, Integrase-Deficient Lentiviral Vector as an All-in-One Platform for Highly Efficient CRISPR/Cas9-Mediated Gene Editing. Mol Ther Methods Clin Dev 5, 153-164 (2017).

67. C. McQuin et al., CellProfiler 3.0: Next-generation image processing for biology. PLoS Biol 16, e2005970 (2018).

68. S. C. Chapin, V. Germain, E. R. Dufresne, Automated trapping, assembly, and sorting with holographic optical tweezers. Opt Express 14, 13095-13100 (2006).

69. D. Montiel, H. Cang, H. Yang, Quantitative characterization of changes in dynamical behavior for single-particle tracking studies. J Phys Chem B 110, 19763-19770 (2006).

70. L. Horvath, The Maximum Likelihood Method for Testing Changes in the Parameters of Normal Observations. The Annals of Statistics 21, 671-680 (1993).

71. P. Enkhbayar, S. Damdinsuren, M. Osaki, N. Matsushima, HELFIT: Helix fitting by a total least squares method. Computational Biology and Chemistry 32, 307-310 (2008).

Acknowledgments: The authors acknowledge the Duke Viral Vector Core for assistance with virus-like particle generation and the Duke Cell Culture Facility for access to cell lines used in this study. The authors would also like to thank LSM Tech for help customizing the laser scanning microscope for integration into the 3D-TrIm setup. The authors are also grateful to Duke Office of Information Technology (OIT) and Duke Research Computing for facilitating access to Amira 3D 2021.1 for data rendering. 


\section{Funding:}

National Institutes of Health grant R35GM124868 (KDW)

Duke University (KDW)

\section{Author contributions:}

Conceptualization: CJ, JE, KDW

Methodology: CJ, JE, KDW

Investigation: $\mathrm{CJ}, \mathrm{JE}, \mathrm{YL}$

Data Curation: CJ, JE, YL, JA, KDW

Visualization: CJ, JE, YL, JA, KDW

Funding acquisition: KDW

Writing - original draft: CJ, JE, KDW

Writing - review \& editing: CJ, JE, KDW

Competing interests: Authors declare that they have no competing interests.

Data and materials availability: Code to analyze single virus trajectories (MATLAB) and render them (Amira) is available at https://github.com/welsherlab/3dtrim. Raw data available from the corresponding author upon reasonable request.

\section{Supplementary Materials}

Materials and Methods

Figs. S1 to S38

20 References (61-71)

Movies S1 to S8 


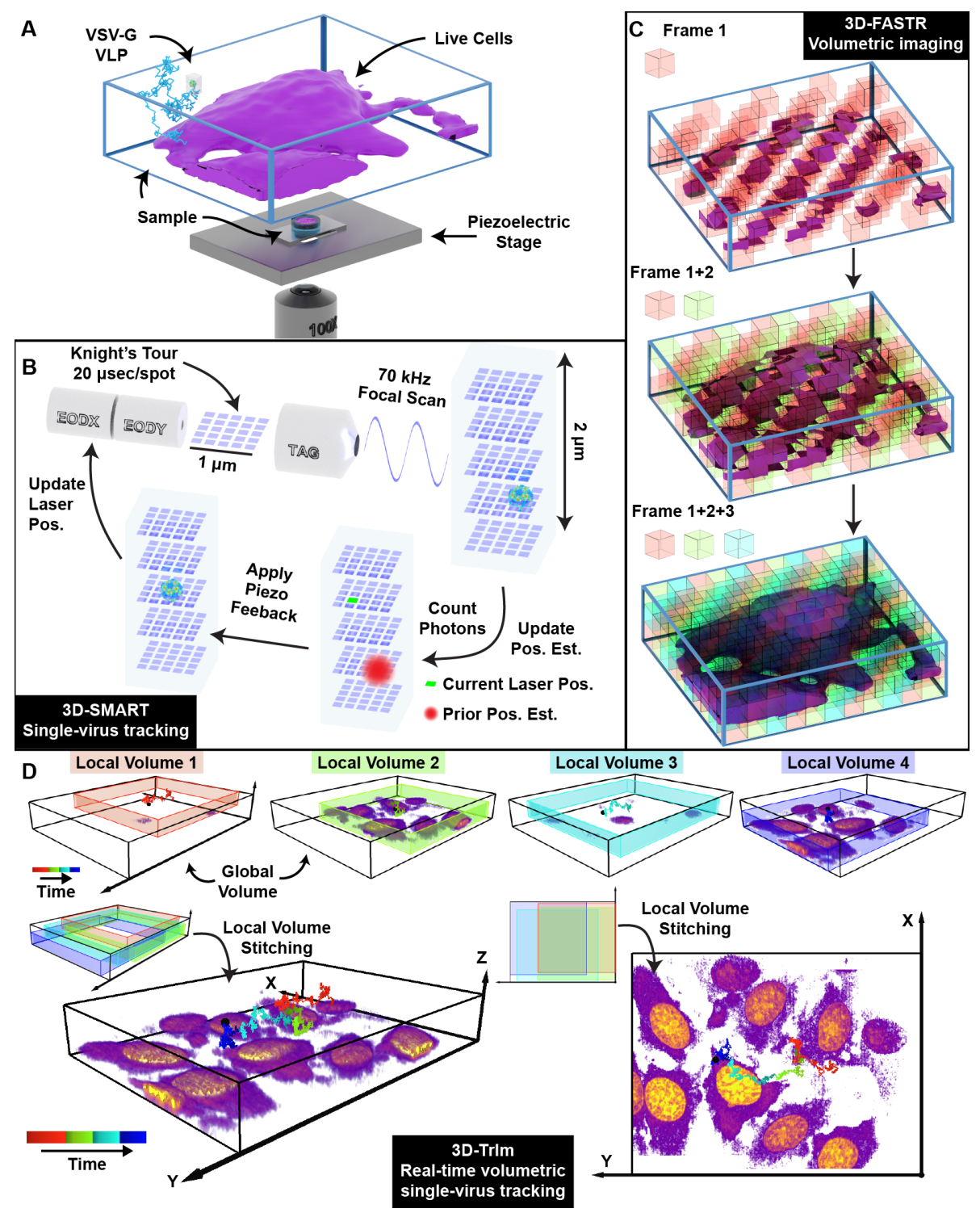

Fig. 1. 3D Tracking and Imaging (3D-TrIm). (A) Experimental setup. Fluorescently labeled VLPs are added to live cells plated on a coverslip. The sample is placed on a heated sample holder mounted on a piezoelectric stage. (B) Overview of 3D-SMART tracking of single viruses. The electro-optic deflector (EOD) and tunable acoustic gradient (TAG) lens rapidly scan the local particle area. Photon arrival times and the current laser position are used to calculate the position of the virus within the scan area. Using the measured position, the piezoelectric stage moves to recenter the virus within the scan area. (C) Concept of 3D-FASTR volumetric imaging. By outfitting a traditional 2-photon LSM with an ETL, a repeatable, tessellated 3D sampling pattern can be generated during each frame-time. Over a set number of frame-times, the entire volume is sampled. (D) Construction of global volumes in 3D-TrIm. As the virus diffuses, 3D-SMART moves the sample, and the 3D-FASTR imaging system collects sequential volumes from different areas around the particle. These time-resolved local volumes can be used to generate an integrated global volume (see also: movie S1). 
bioRxiv preprint doi: https://doi.org/10.1101/2021.12.17.473224; this version posted December 19,2021 . The copyright holder for this preprint

(which was not certified by peer review) is the author/funder, who has granted bioRxiv a license to display the preprint in perpetuity. It is made available under aCC-BY-NC-ND 4.0 International license.
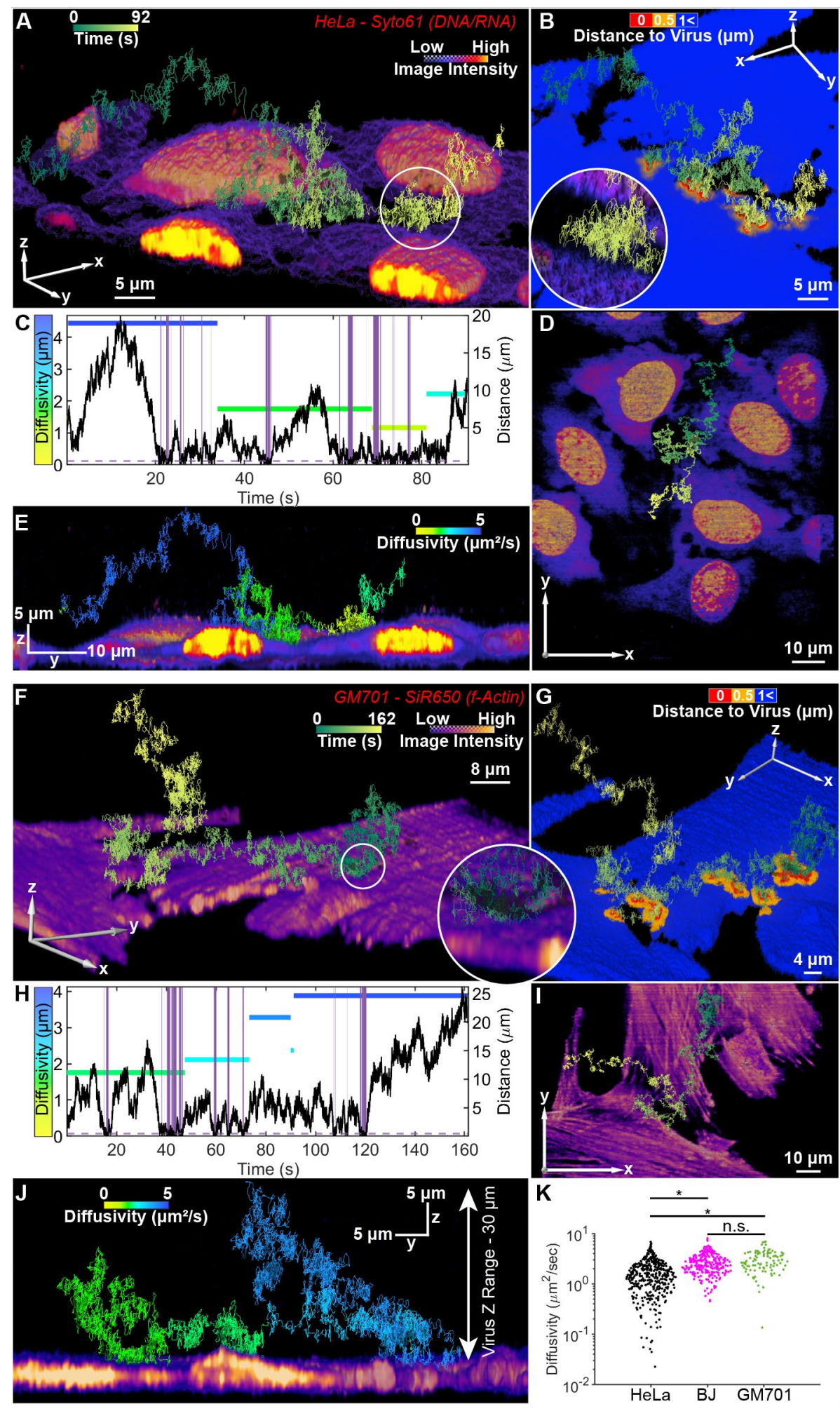

Fig. 2. Minute-long high-resolution 3D tracking and volumetric live-cell imaging reveals transient VSV-G VLP contacts with the cell surface. (A and B) 3D reconstruction of a single 
VSV-G VLP trajectory with live HeLa cells from a 4D data set. Cells are color-coded by intensity and distance from the cell surface to the virus trajectory in (A and B), respectively. Circular inset: enlarged view of "skimming" event. (C) Correlation between diffusivity and cell-to-virus distance. Skimming event shown in circular inset of (B) occurs between 68-78 sec. (D) Top-down (xy) complete imaging area with trajectory. (E) Lateral (yz) view with trajectory color-coded by diffusion coefficient segments calculated by change-point analysis. (F-J) Identical to (A-E) except VSV-G trajectory co-registered with live GM701 fibroblast cells (movie S2). Skimming event shown in circular inset of (F) occurs between 37.5-47.5 sec. (K) Diffusivity of VSV-G VLP trajectory segments identified by change-point analysis. (HeLa, BJ, and GM701 n = 413, 255, and 100 segments, from 66,105 , and 47 trajectories, respectively). Statistical significance was assessed by 2 -sample $t$-test, on the $\mathrm{p}$-value at $\mathrm{p}<0.01$. 

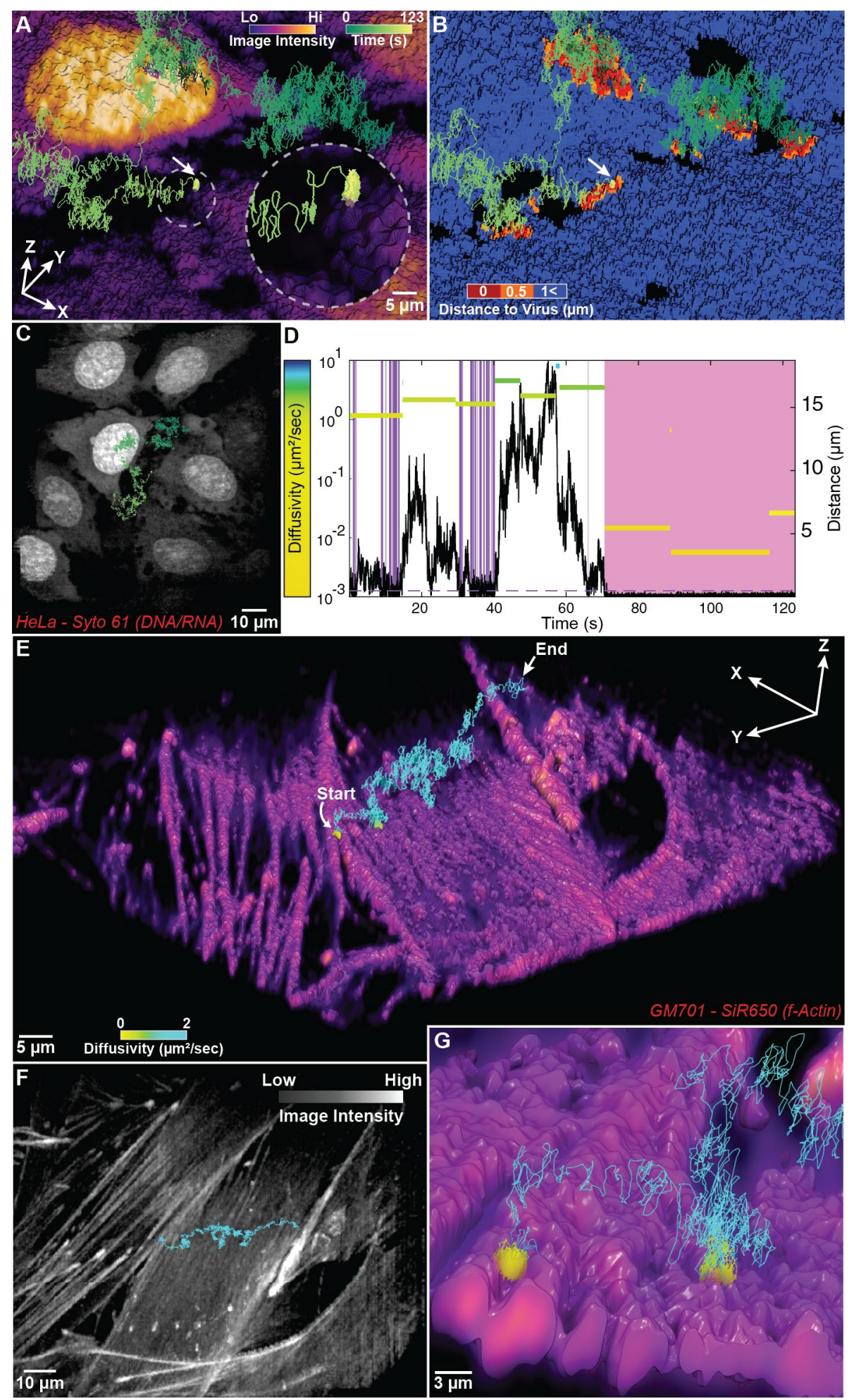

Fig. 3. VSV-G Binding. (A and B) 3D TrIm render of live nucleic acid-labeled HeLa cells. (A) Intensity volume render (see also: movie S3). Grey circular inset shows moment of binding. (B) Virus-Cell distance render. (C) Maximum intensity projection (MIP) with trajectory overlay. (D) Trajectory diffusivity and virus-cell distance time trace. (E-G) 3D TrIm render of live actin- 
bioRxiv preprint doi: https://doi.org/10.1101/2021.12.17.473224; this version posted December 19, 2021. The copyright holder for this preprint (which was not certified by peer review) is the author/funder, who has granted bioRxiv a license to display the preprint in perpetuity. It is made available under aCC-BY-NC-ND 4.0 International license.

labeled GM701 cells with trajectory color-coded by diffusivity (see also: movie S4). (F) MIP with trajectory overlay. (G) Close-in view of VLP binding sites. 

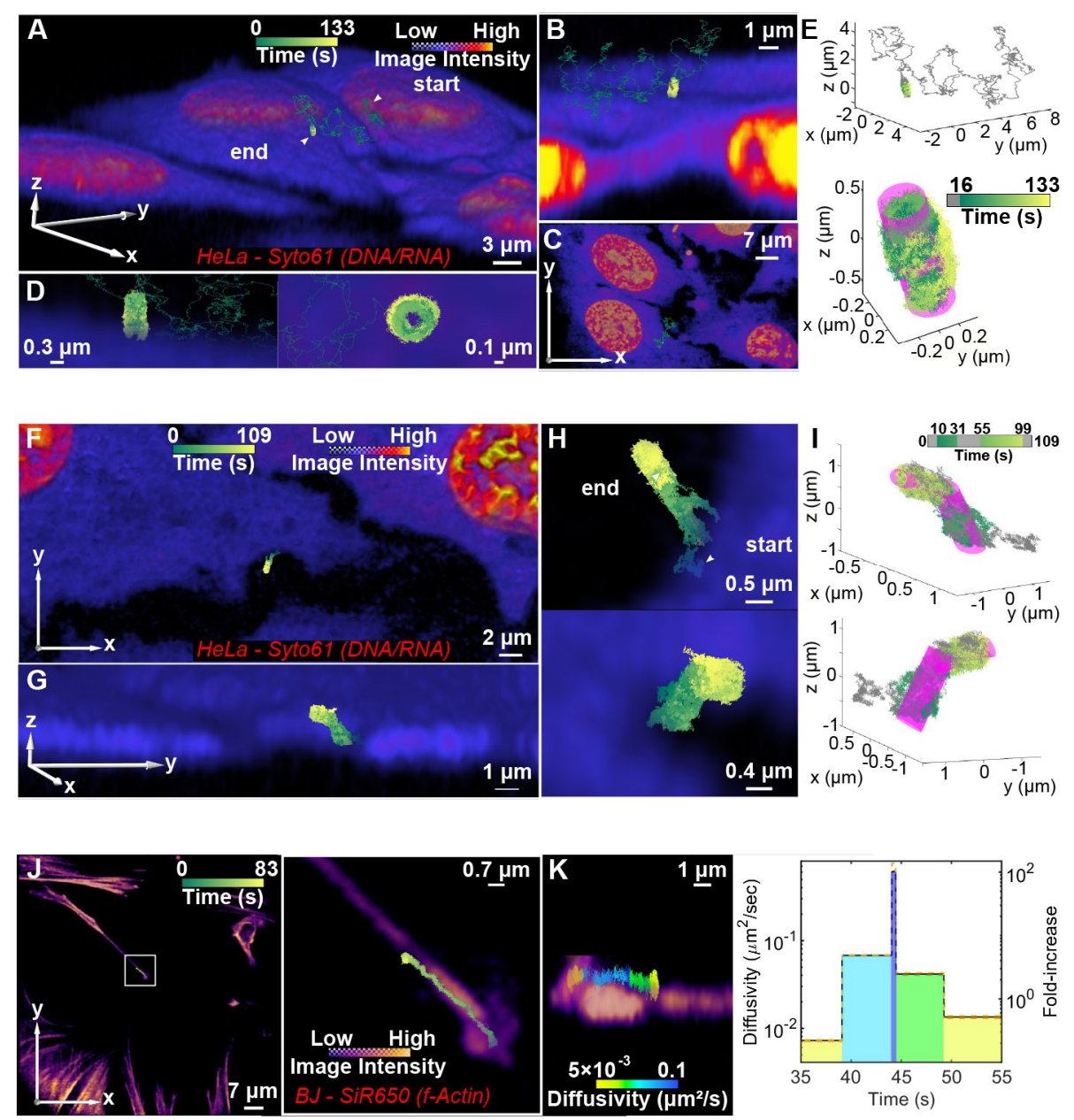

Fig. 4. Virus interaction with filopodia. (A) VSV-G landing on filopodium protruding from the surface of a live cell (see also: movie S5). (B) Exploded view with slices through $\mathrm{x}$ and y axes. (C) Top-down (xy) complete imaging area with trajectory. (D) Magnified views showing the hollow cylinder the VLP draws out while diffusing around the filopodium. (E) Cylindrical fitting of the bound portion of the trajectory in (A-D) $(16-133 \mathrm{sec})$, radius $=105 \pm 8.4 \mathrm{~nm}$. (F) Additional high-resolution trajectory of VLP on a filopodium captured after landing. (G) Lateral view, VLP tracking captures change in filopodium shape. (H) Enlarged views showing VLP progression after starting from a proximal position on the membrane. (I) Cylindrical fitting of base (10-31 sec) and extremity $(55-99 \mathrm{sec})$ of the trajectory in $(\mathrm{F}-\mathrm{H})$, radius $=153 \pm 8.7 \mathrm{~nm}$ and $77 \pm 8.4 \mathrm{~nm}$, respectively. Average filopodium radius of $125 \pm 16 \mathrm{~nm}(\mathrm{n}=5)$. (J) Viral surfing along filopodium towards main body of cell. Inset: enlarged view at tip of protrusion. (K) Left: lateral view, with trajectory color-coded by diffusion coefficient segments calculated by change-point analysis. Right: Time course showing significant change in diffusivity as linear motion proceeds. Dashed lines represent SD. 

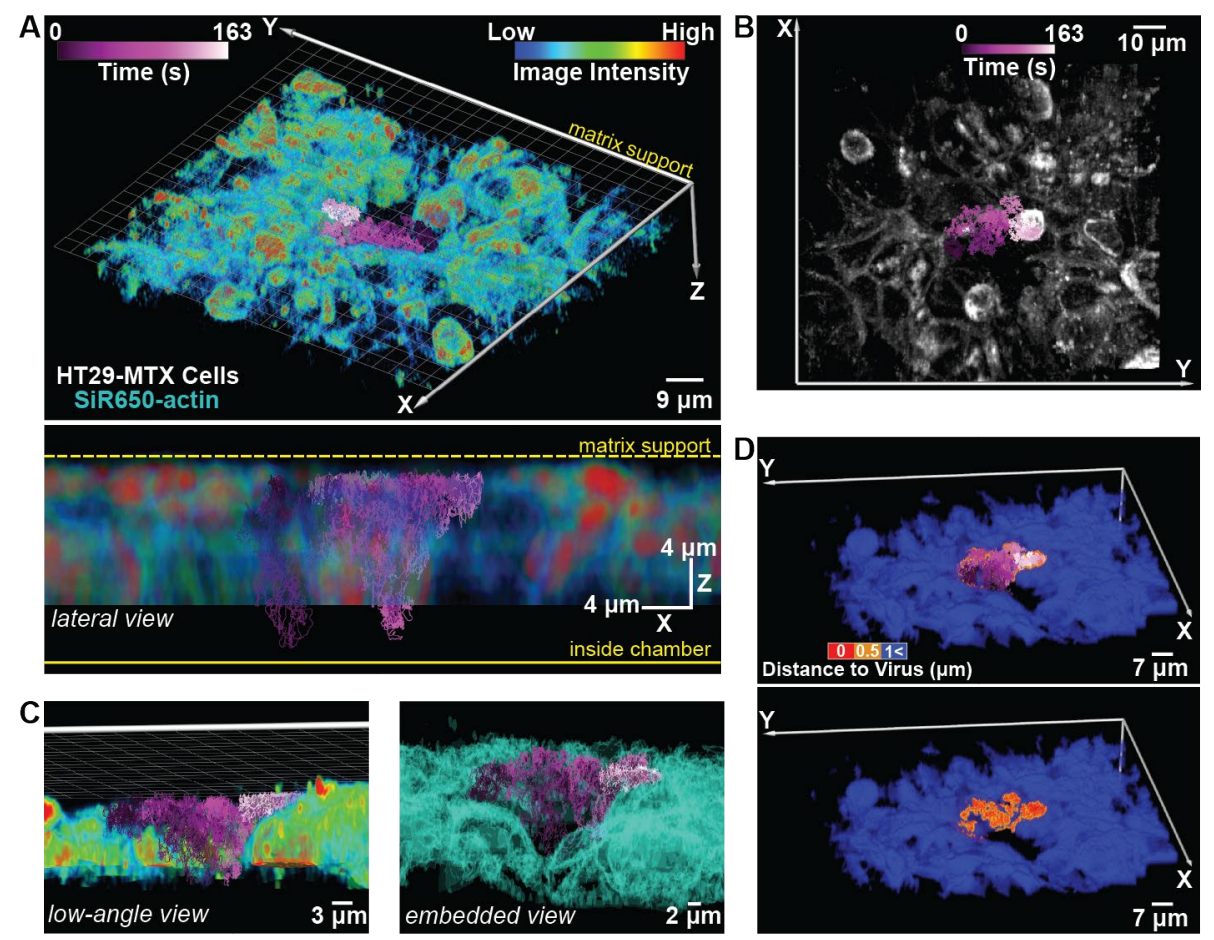

Fig. 5. VSV-G VLP diffusing through multi-layered epithelial cells. (A) Top: 3D reconstruction from a 4D data set covering 10 local volumes, at 10 FPV (see also: movie S6) of suspended HT29-MTX cells grown on inverted matrix support. Co-registered VSV-G VLP trajectory color-coded by time. Bottom: XZ view. (B) Top-down (xy) MIP. (C) Right: magnified cut-through view showing VSV-G VLP confined within vacancy as it diffuses around the edge of a cell. Left: Isosurface render of cells highlighting sample density. (D) Top: the distance of the trajectory to the cell surface is projected as a distance map on the cell volume, with the trajectory color-coded by time overlayed. Bottom: Volume with trajectory omitted, showing areas close contact on the cell surface. 


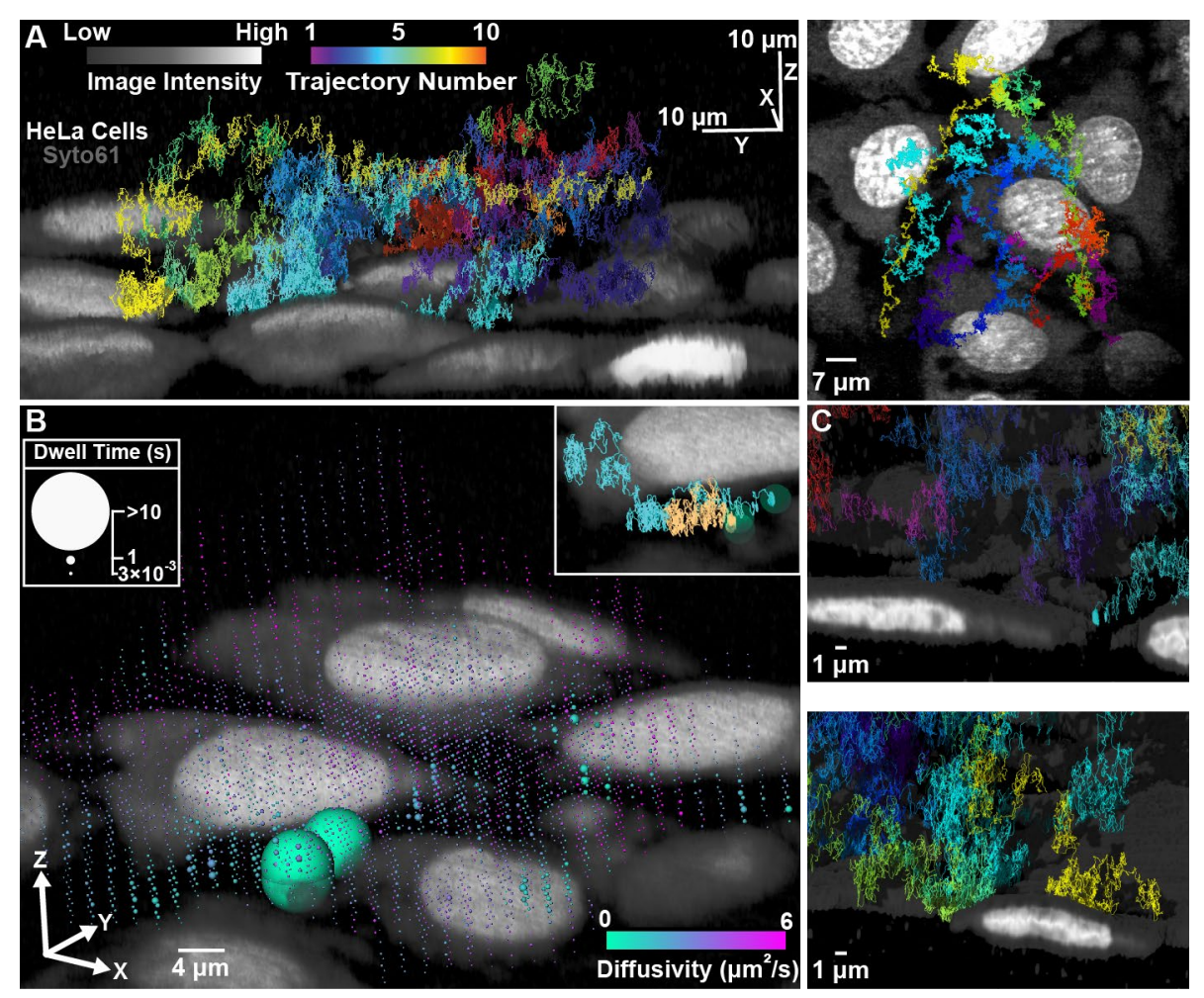

Fig. 6. Multi-trajectory acquisition. (A) Left: 10 VSV-G VLP trajectories collected in the same area of live HeLa cells stained with SYTO61 (see also: fig. S38 and movie S7). Right: top-down (xy) MIP with multiple co-registered VLP trajectories. Colorbar represents trajectory number, with each trajectory uniquely colored. (B) The dwell time of 27 VSV-G VLP trajectories (starting from free diffusion) collected in the same area as (A) were binned into voxels and represented as spheres, color-mapped by diffusivity. This correlated analysis reveals commonly visited regions and highlights the slower diffusion experienced by the VLP population nearer the cell surface. Inset: two different VLPs land in close proximity on the same cell. (C) Exploded views showing multiple VLPs contact the same cell. 